Check for updates

Cite this: RSC Adv., 2019, 9, 33187

Received 14th August 2019

Accepted 8th October 2019

DOI: $10.1039 /$ c9ra06364a

rsc.li/rsc-advances

\section{Gel actuators based on polymeric radicals $\uparrow$}

\author{
Ravindra N. Wickramasinhage, (D) a Shailesh K. Goswami, ${ }^{a}$ C. John McAdam, ${ }^{a}$ \\ Sharali Malik, (D) ${ }^{\text {b }}$ Lyall R. Hanton (D) ${ }^{\text {a }}$ and Stephen C. Moratti (D) *a
}

Low-voltage electrochemical actuation of radical polymer gels has been demonstrated in an organic electrolyte. Polymer gels were prepared by post-modification of active-ester precursor gels with an amine-functionalised radical. A combination of few-layer graphene and multiwall carbon nanotubes gave high conductivity and improved actuation in the gels, with 32\% linear actuation. The actuator system showed good stability over at least 10 cycles, showing its promise. The cycle time was several hours due to mass-transport limited transport of ions and solvent into the device.

\section{Introduction}

Commercial solid-state actuators include shape memory alloys and piezoelectric ceramics. Those inorganic materials are typically heavy, nonflexible and relatively expensive. Shape memory alloys require high temperature while electroactive piezoelectric ceramic need high electric fields but only produce a small strain. Since the 1990s, polymer based electroactive materials (EAPs) have emerged as potential components of actuators..$^{1-3}$ EAPs have useful properties such as low density, high flexibility, good fracture tolerance, potentially large strains and low manufacturing cost.

Conducting polymers (CPs) form a subgroup of EAPs, ${ }^{1,2}$ and have some unique advantages in this application. For example, conducting polymers show a catch state, ${ }^{4}$ (i.e. only a minimal amount of energy is needed to hold a steady load), high work density, ${ }^{5}$ moderate to high strain (1-40\%), moderate stress, and good flexibility. CPs undergo volumetric changes when switching between their oxidised and reduced state and this can be exploited to perform work..$^{4,6,7}$ This volume change is only small (up to a few percent) so to achieve high strains CP actuators are employed in bending or twisting modes, which limits the achievable stress values. Work density is the amount of work generated in one actuator cycle per actuator volume and values for CPs can be within a factor of four as compared to combustion engines. ${ }^{\mathbf{1}}$ Low voltage operations accompanied with high work densities make CPs attractive in applications such as medical devices and microfluidics. ${ }^{5}$

CPs can be divided into groups, including conjugated polymers (e.g. polypyrrole, polyaniline, polyacetylene etc.) and redox

${ }^{a}$ Chemistry Department, University of Otago, Dunedin, New Zealand. E-mail: smoratti@chemistry.otago.ac.nz

${ }^{b}$ Karlsruhe Institute of Technology (KIT), Institute of Nanotechnology, D-76131 Karlsruhe, Germany

$\dagger$ Electronic supplementary information (ESI) available. See DOI: 10.1039/c9ra06364a polymers (RPs). Redox polymers contain unconjugated backbones and covalently attached redox sites, either built into the chain, or as pendent groups. ${ }^{8,9}$ RPs need to have a high density of redox active groups in the polymer backbone to be (semi) conducting, to allow charge transport via hopping.

Previously we showed that a quinone-containing polymer could be usefully employed in a gel actuator. ${ }^{10}$ The operation of the actuator was limited by the poor conductivity of the gel. However, substantial improvement was found by incorporating carbon nanotubes into the material. The gel swelled upon reduction of the quinone units into negatively charged phenolic units in the basic organic medium. However, side-reactions during redox cycling led to solvent and functional group degradation, and eventual loss of action. This necessitated changing the solvent after several cycles to keep the device performing. The basic nature of the solvent may have also contributed to hydrolysis of the cross-linkers and functional groups. Despite this, the device showed good linear expansion of $c a .40 \%$ at low potential $\left(+1 \mathrm{~V} v s . \mathrm{Fc} / \mathrm{Fc}^{+}\right)$.

In this work, we utilise a class of pendent polymers that have also been investigated for use in battery technology. ${ }^{11-15}$ These RPs bear pendant, stable radical groups attached to the polymer backbone. ${ }^{12-14}$ 2,2,6,6-Tetramethylpiperidinoxy (TEMPO) is the most common pendent group used in radical polymers due to its excellent stability, and ease of synthesis and incorporation. Cyclic voltammetry (CV) of TEMPO in water reveals a reversible oxidation wave at $0.49 \mathrm{~V}$ on the anodic side but irreversible redox behaviour at $-0.62 \mathrm{~V}$ on the cathodic side ( $v s$. a saturated calomel electrode at $\mathrm{pH} 7) .{ }^{16}$ The reversible one electron oxidation process from the TEMPO radical to the oxoammonium salt $\left(\mathrm{TEMPO}^{+}\right)$is illustrated in Scheme 1. This redox system has been shown to be stable over many hundreds of cycles in batteries, ${ }^{\mathbf{1 1 - 1 4}}$ and so looked to be a promising candidate for use in actuators. The oxidised form of TEMPO is a potent oxidizing agent, which for instance will rapidly oxidise alcohols into their corresponding aldehydes. ${ }^{17,18}$ This limits the 
range of solvents and comonomers that can be used with the system.

\section{Experimental}

\section{Materials}

Dicyclohexyl carbodiimide (DCC) (538-75-0), methacrylic acid (79-41-4), $N$-hydroxysuccinimide (97\%) (6066-82-6), 4-amino2,2,6,6-tetramethylpiperidine (98\%) (36768-62-4), acetic anhydride $\left(\mathrm{Ac}_{2} \mathrm{O}\right)$ (108-24-7), sodium carbonate anhydrous (497-198), sodium tungstate dihydrate $\left(\mathrm{Na}_{2} \mathrm{WO}_{4}\right)$ (10213-10-2), hydrogen peroxide (30\%) (7722-84-1), and 1,1'-azobis(cyclohexanecarbonitrile) (ABCN) (2094-98-6) were used as received (Sigma-Aldrich). MWCNT were obtained from Nanocyl (Nanocyl 3100 , diameter $9.5 \mathrm{~nm}$, length $1500 \mathrm{~nm}){ }^{19}$ Few layered graphene (FLG) was obtained through heat treatment of multilayered graphene. ${ }^{20}$ Styryl-functionalised star macro-cross linker (S-XL) was synthesised according to the literature. ${ }^{21}$

\section{Synthesis of $N$-hydroxysuccinimide methacrylate $(1)^{22}$}

DCC $(8.7 \mathrm{~g}, 42.1 \mathrm{mmol})$ in ethyl acetate $(20 \mathrm{~mL})$ was added dropwise to a solution of methacrylic acid (2.4 g, $27.4 \mathrm{mmol})$ and $N$-hydroxysuccinimide $(5.0 \mathrm{~g}, 42.1 \mathrm{mmol})$ in ethyl acetate $(25 \mathrm{~mL})$ at $0{ }^{\circ} \mathrm{C}$. The mixture was stirred overnight at room temperature, the resulting suspension was filtered, and the solvent was removed from the filtrate under reduced pressure, to obtain a white solid. This was purified by column chromatography using EtOAc/PET (30: 70) over silica.

Yield 75\%; Anal Calcd for $\mathrm{C}_{8} \mathrm{H}_{9} \mathrm{NO}_{4}$ : C, 52.46; $\mathrm{H}, 4.95 ; \mathrm{N}$, 7.65; found: C, 52.54; H, 5.01; N, 7.76; ${ }^{1} \mathrm{H}$ NMR $(400 \mathrm{MHz}$, $\left.\mathrm{CDCl}_{3}\right): \delta 6.39(\mathrm{q}, J=1.0 \mathrm{~Hz}, 1 \mathrm{H}), 5.86(\mathrm{dt}, J=2.3,1.1 \mathrm{~Hz}, 1 \mathrm{H})$, $2.84(\mathrm{~s}, 4 \mathrm{H}), 2.05(\mathrm{~s}, 3 \mathrm{H}) ;{ }^{13} \mathrm{C} \mathrm{NMR}\left(101 \mathrm{MHz}, \mathrm{CDCl}_{3}\right): \delta 169.2$, 162.1, 131.9, 130.4, 25.6, 18.2.

\section{Preparation of nanocarbon dispersion}

DMF (10 mL, $9.48 \mathrm{~g}$ ) was mixed with $50 \mathrm{mg}$ of MWCNT, and $15 \mathrm{mg}$ of few layered graphene (FLG). The mixture was sonicated for $4 \mathrm{~h}$ in a Bandelin RK 52 ultrasonic bath, keeping the temperature range between $5-10{ }^{\circ} \mathrm{C}$ to avoid overheating. To this dispersion was added another $50 \mathrm{mg}$ of MWCNT and $15 \mathrm{mg}$ of FLG and it was sonicated for another $3 \mathrm{~h}$ at the same temperature range. The final dispersion contained MWCNT/FLG in $10: 3$ weight ratios and $1.35 \%(\mathrm{w} / \mathrm{w})$ in solids. The total amount of each component in the final gels is detailed in Table S1 (ESI $\dagger$ ).

\section{Preparation of actuators}

A plastic mould was produced by melting the exit end of a $1 \mathrm{~mL}$ plastic syringe (inner diameter of $\sim 4.7 \mathrm{~mm}$ ). A coiled Pt wire electrode was constructed with 12 turns (wire thickness 0.0127 $\mathrm{mm}$ ); with an overall coil length of $1 \mathrm{~cm}$ and a coil diameter of $2.5 \mathrm{~mm}$. Enough wire $(\mathrm{ca} .2 \mathrm{~cm})$ was left at one end of the coil to allow electrical connection.

The Pt coil was placed inside the plastic syringe mould. A mixture of monomer 1 (400 mg), crosslinker S-XL (20 mg), ABCN $(30 \mathrm{mg})$ and nanocarbon dispersion $(1.40 \mathrm{~g})$ was degassed by bubbling argon for 5 minutes, and added into the syringe/coil assembly. The top was sealed and polymerized at $70{ }^{\circ} \mathrm{C} \mathrm{o} / \mathrm{n}$. The polymer gel was removed from the syringe and soaked in a solution of 4-amino-TEMPO (5 equivalents) in DMF-dioxane $(70: 30, \mathrm{v} / \mathrm{v})$ at room temperature for 4 days. The resulting radical gel composite is termed G2-C; the equivalent gel without the nano-carbon dispersion is termed G2. The gels were then put into DMF-MeCN (10/90 v/v) containing $0.1 \mathrm{M} \mathrm{NBu}_{4} \mathrm{PF}_{6},(20$ $\mathrm{mL}$ ) for 1 day to extract any unreacted amine and to ready the gel for actuation. For actuation measurements the gel was cycled between $+0.5 \mathrm{~V}$ and $-1.04 \mathrm{~V}$ against an internal reference of ferrocene $\left(\mathrm{Fc} / \mathrm{Fc}^{+}\right)$in the above electrolyte system.

\section{Cyclic voltammetry}

Cyclic voltammetric (CV) experiments were performed at $20{ }^{\circ} \mathrm{C}$ in DMF-MeCN $(10 / 90 \mathrm{v} / \mathrm{v})$ with $0.1 \mathrm{MBu}_{4} \mathrm{NPF}_{6}$ as the supporting electrolyte, degassed with argon. A three-electrode cell was used with a BAS $3.0 \mathrm{~mm}$ diameter glassy carbon working, platinum wire auxiliary and reference electrodes. A $3 \times 3 \times 0.5 \mathrm{~mm}$ slice of gel (either G2 or G2-C) was placed on the working electrode surface and retained with dialysis membrane and a rubber ring. Voltammograms were recorded with the aid of a Powerlab/4sp computer-controlled potentiostat with internal reference of ferrocene $\left(\mathrm{Fc} / \mathrm{Fc}^{+}\right)$.

\section{Results \& discussion}

Three approaches were attempted for the preparation of TEMPO-containing gels. Direct polymerisation of a methacrylated nitronium salt has been demonstrated, ${ }^{23-25}$ but any traces of residual TEMPO-containing monomer will inhibit the polymerisation completely. We found it impossible to prepare the monomer salt free of any radical impurities, and so this route was abandoned. It is also possible to polymerise a respective
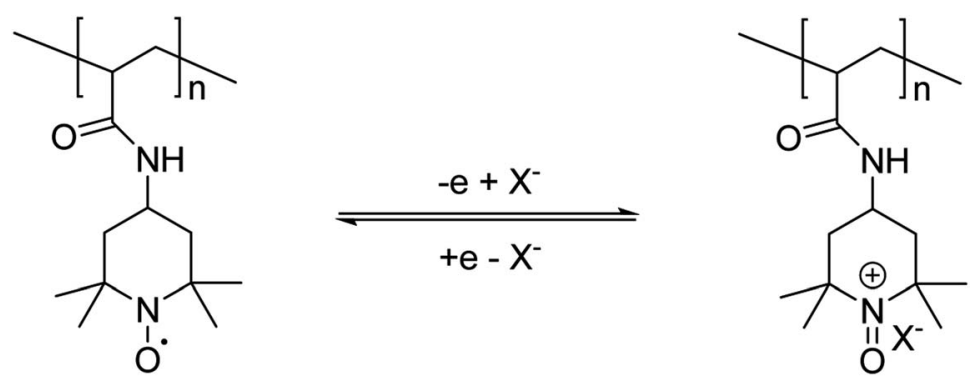

Scheme 1 The reversible redox process in a polymer-based TEMPO/TEMPO ${ }^{+}$couple. 
piperidine monomer and oxidise the amine groups afterwards with $m$-chloroperbenzoic acid (mCPBA). ${ }^{\mathbf{1 4}}$ However, actuator performance was not as good as desired, and the actuators failed after one cycle. This may be due to over-oxidation of the piperidine units which is a known side-reaction of this oxidation route. ${ }^{26}$ The best method proved to be radical polymerisation of an active ester monomer together with a starmacrocross-linker ${ }^{\mathbf{1 0}}$ to form a gel G1. The monomer incorporation appears to be quantitative as no residual monomer can be extracted after soaking in excess solvent. This polymerization is followed by nucleophilic substitution with a nitroxidecontaining amine (Scheme 2) to give a radical containing gel G2. Infrared analysis of the gels during substitution showed that 4 days reaction was needed at $\mathrm{rt}$ for the active ester peak in the IR spectra at $1727 \mathrm{~cm}^{-1}$ in gel 2 to largely disappear and to be replaced by an amide peak at $1647 \mathrm{~cm}^{-1}$ (ESI $\dagger$ ).

We have found previously that it is necessary to incorporate conductive fillers in these actuators as the gels are not conductive enough by themselves. ${ }^{10}$ Indeed the same was found in the present study, that no actuation was seen in the pristine gels without added conductive fillers. We also find here that no actuation is produced without the electroactive TEMPO radical being present (e.g. using G1 or G1-C). The previous work used multiwalled carbon nanotubes (MWCNTs) dispersed with poly(vinyl pyridine) (PVP). We were interested to see whether the more conductive graphene could also be used. The best dispersion results were obtained with a $3: 1 \mathrm{w} / \mathrm{w}$ mixture of MWCNTs and few-layer graphene (FLG) (sonicated at low temperatures to prevent damage to the nanotubes ${ }^{27}$ ). Both these needed a dispersing agent such as PVP to stop sedimentation and aggregation when used alone in organic solvents. However when used together stable suspensions were formed without the use of any other agents, as they appeared to interact with each other and help prevent flocculation and sedimentation. As shown in the TEM image of the resulting carbon-containing gel G2-C, (Fig. 1) the FLG in this gel is not well connected with each other due to its low concentration, but the nanotubes appear to bridge the gaps, allowing for a good electrical conducting network to be formed. It has been noted by others that mixtures of carbon nanotubes and graphene are often more conductive at low loading levels than either by themselves. ${ }^{28}$

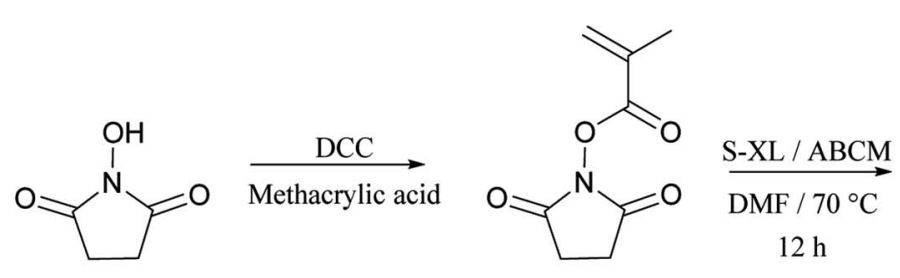

N-hydroxysuccinimide

(1) $75 \%$<smiles>CC(C)(C)CC(C)(C)C(=O)ON1C(=O)CCC1=O</smiles>

G1

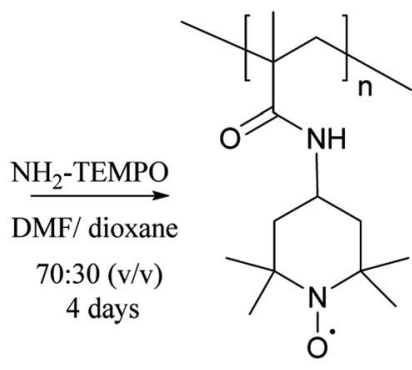

G2

Scheme 2 Post-modification of active-ester precursor gels with an amine-functionalised radical in the synthesis of the actuator gels.
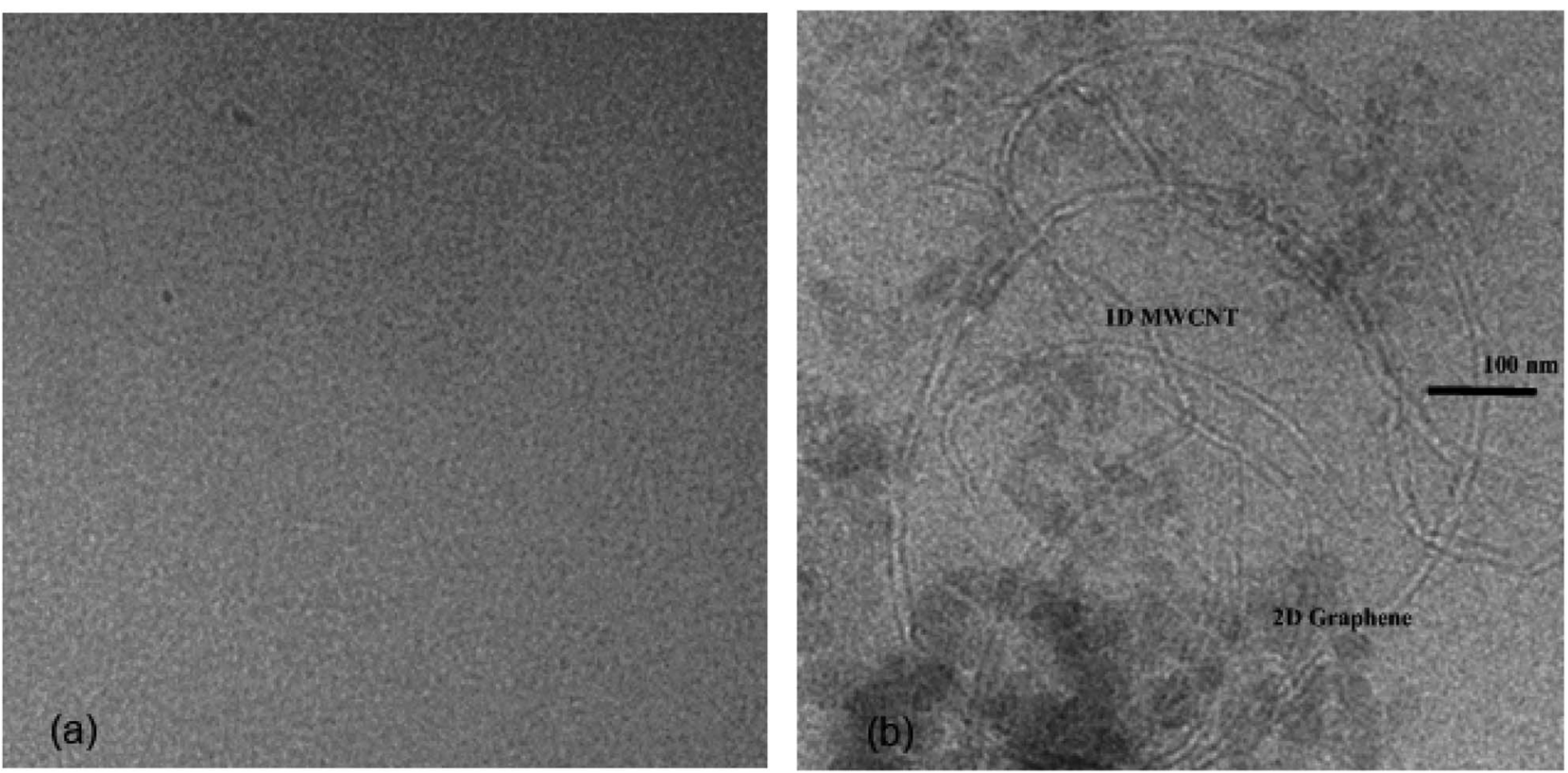

Fig. 1 TEM image of (a) gel G2 without and (b) G2-C with MWCNTs and FLGs (both to same scale). 
The gel G2 (without carbon fillers) shows the typical CV expected for a TEMPO radical, confirming successful post modification (Fig. 2a). The equivalent gel sample G2-C, containing the dispersed nanocarbons, also showed the respective oxidation and reduction peaks but with wider peak-to-peak separation and with a much higher peak current (Fig. 2b). The difference was that G2 was poorly conductive, and hence reversible oxidation and reduction only occurred close to the electrode surface. The conductive carbon filler allowed redox processes to occur throughout the gel thickness, increasing the peak current. This large current required the equivalent movement of large amounts of ions through the gel which was slow, hence resulting in the broadening and shifting of the redox peaks. The poor conductivity of $\mathbf{G} 2$ also meant that no actuation was seen, unlike G2-C.

Actuators were constructed using a platinum coil electrode embedded into the gel. The coil allowed electrical contact, as well as promoting linear expansion of the gel. ${ }^{10}$ The gel-coil composite was cycled between $+0.5 \mathrm{~V}$ and $-1.04 \mathrm{~V}$ against internal reference of ferrocene $\left(\mathrm{Fc} / \mathrm{Fc}^{+}\right)$in electrolyte system of DMF-MeCN $(10 / 90 \mathrm{v} / \mathrm{v})$ containing $0.1 \mathrm{M} \mathrm{NBu}_{4} \mathrm{PF}_{6}$ as electrolyte. This solvent mix was found to give the best performance of the solvents tried, and produced a large difference in the swelling of the gel between the oxidised and reduced states. Other electrolytes were tested, including $\mathrm{LiClO}_{4}, \mathrm{LiOAc}_{2} \mathrm{Et}_{4} \mathrm{NBF}_{4}, \mathrm{Et}_{4} \mathrm{NPF}_{6}$, and $\mathrm{NBu}_{4} \mathrm{BF}_{4}$, however the actuation rate was much slower and so these were not examined further. The reason for effect this is not clear, but it might be that the larger size of the $\mathrm{NBu}_{4} \mathrm{PF}_{6}$ ions produces the largest swelling effect and thus apparent actuation rate. While the reduction voltage represented a substantial overpotential over what might be expected to be needed ( $c a .0 .2$ $\mathrm{V}$ ), it ensured that the expansion and contraction occurred at similar rates. Lower voltages produced a much slower contraction, possibly due to the expanded gel being less conductive. The gel expanded linearly by about $32 \%$ in 90 minutes, and shrunk back to its original state after reduction in a similar period of time (Fig. 3). Even further expansion appeared possible from the extension versus time graph (Fig. 4), but the gels were somewhat fragile when expanded and prone to tearing on prolonged oxidation. While our previous gel showed slightly larger expansion $(40 \%),{ }^{10}$ it is still much better than previously reported conducting polymer actuators, which only show strains of a few percent on redox cycling.

This system was tested for 10 cycles (Fig. 4) with little change in performance without any change of solvent. This is in stark contrast to the quinone actuator, ${ }^{\mathbf{1 0}}$ which needed replacement of solvent every few cycles due to unwanted electrode reactions, as evidenced by bubbling from the gel and halting of the actuation.

There remain several issues that can be improved. The actuation is slow, and this might be improved through nanostructuring of the gel, allowing faster transport of solvent and ions through the bulk. Alternatively, the actuator could be miniaturized, which would proportionally speed up the bulk diffusion. To use in practice, the actuator will need to be self-contained to hold the solvent, or used in applications (such as microfluidics) where the solvent is present. However, the performance and stability of the actuator looks very promising.

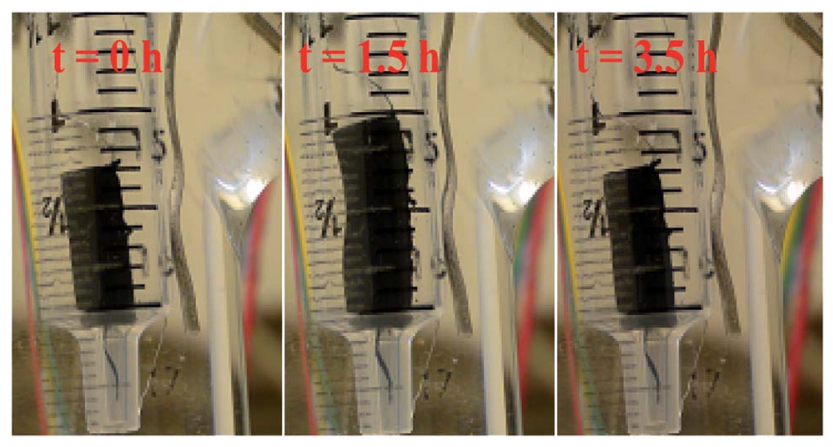

Fig. 3 First actuation cycle of G2-C, in DMF-MeCN (10/90 v/v), $0.1 \mathrm{M}$ $\mathrm{NBu}_{4} \mathrm{PF}_{6}$, from $+0.5 \mathrm{~V}$ and reduction at $-1.04 \mathrm{~V}$ vs. $\mathrm{Fc} / \mathrm{Fc}^{+}$(Video of actuation is included in the ESI $\dagger$ ).
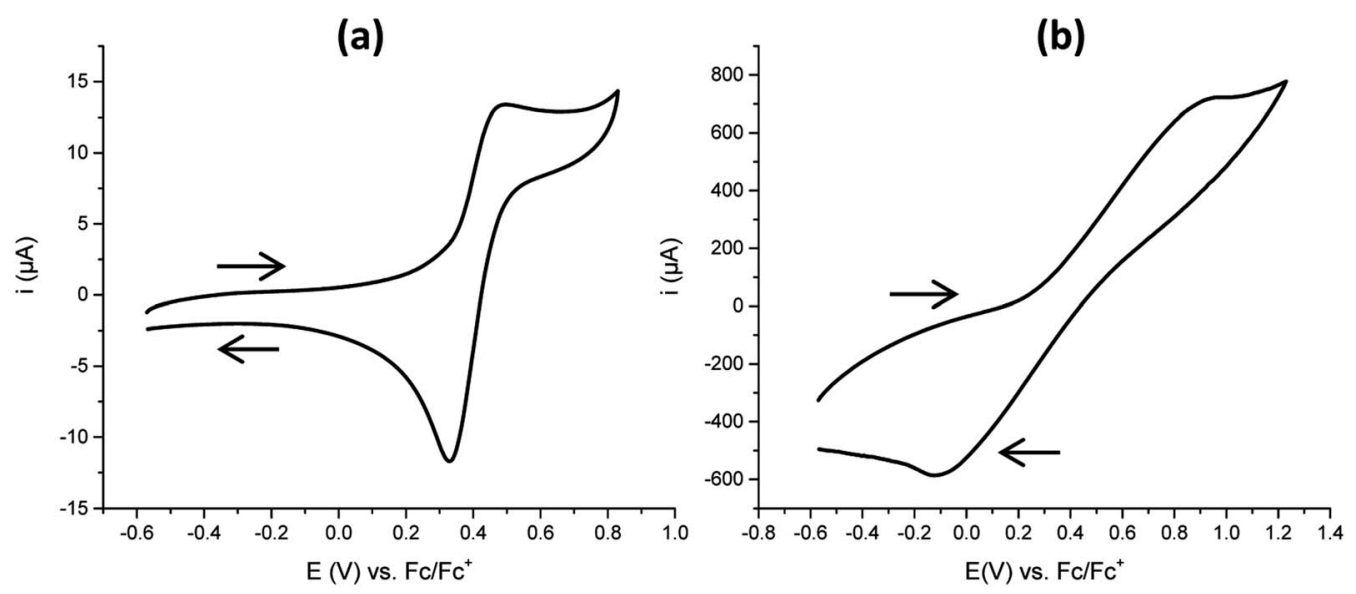

Fig. 2 CVs of homopolymer gel samples obtained by post-modification, (a) CV of post-modified gel without conductive fillers (G2), (b) CV of homopolymer gel contain conductive fillers (G2-C), at the scan rate of $100 \mathrm{mV} \mathrm{s}^{-1}$ in organic solvent mixture of DMF/MeCN (10:90) with $0.1 \mathrm{M}$ of $\mathrm{NBu}_{4} \mathrm{PF}_{6}$ as electrolyte against $\mathrm{Fc} / \mathrm{Fc}^{+}$on a glassy carbon working electrode, Pt reference and Pt auxiliary electrode. 


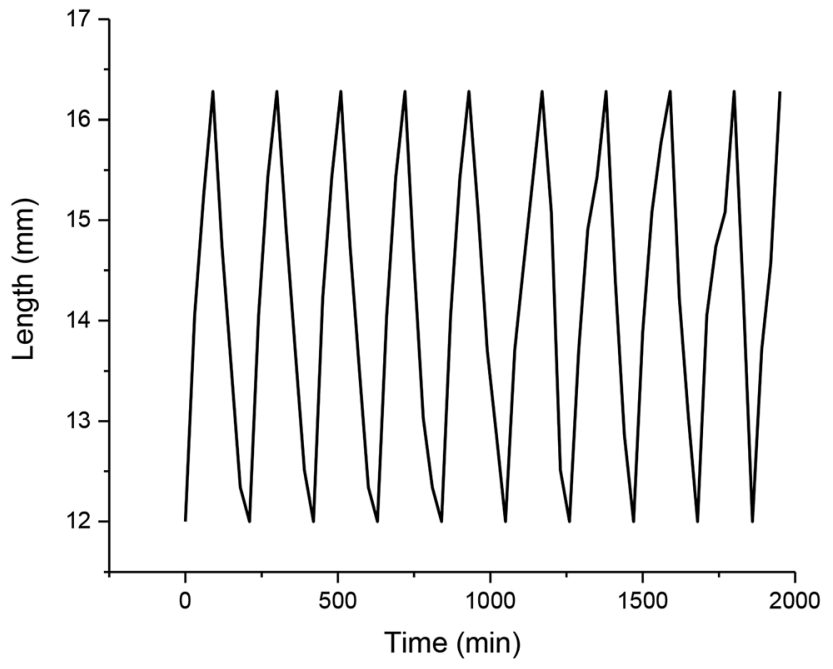

Fig. 4 Extension versus time of the first 10 actuation cycles of the redox gel of G2-C. Oxidation at $+0.5 \mathrm{~V}$ and reduction at $-1.04 \mathrm{~V}$ against internal reference of ferrocene $\left(\mathrm{Fc} / \mathrm{FC}^{+}\right)$.

\section{Conclusions}

A stable actuation system was constructed by modification of an active-ester gel containing conducting nanocarbons with a stable free radical. Stable dispersions of MWCNTs without dispersants can be achieved in the presence of FLG allowing conductivity and improved actuation in the gels. At least $32 \%$ linear actuation was demonstrated over 10 cycles, with a cycle time of 3 hours. No deterioration in performance due to solvent degradation was noted, unlike our previous quinine base actuators.

\section{Conflicts of interest}

There are no conflicts to declare.

\section{Acknowledgements}

The program was supported by the Ministry of Business, Innovation and Employment Science, New Zealand (Project no. UOO-X0808)). S. M. acknowledges support by the Helmholtz Society through program Science and Technology of Nanosystems (STN). The authors would like to acknowledge networking support by the COST Action MultiComp (CA15107), supported by the COST Association (European Cooperation in Science and Technology.

\section{References}

1 R. Samatham, K. J. Kim, D. Dogruer, H. R. Choi, M. Konyo, J. D. Madden, Y. Nakabo, J.-D. Nam, J. Su, S. Tadokoro, W. Yim and M. Yamakita, Active Polymers: An Overview, in Electroactive Polymers for Robotic Applications: Artificial Muscles and Sensors, ed. Kim K. J. and Tadokoro S., Springer London, London, 2007, pp. 1-36.
2 Y. Bar-Cohen, Electroactive Polymers as Artificial Muscles Reality and Challenges, 2001.

3 T. Shiga, Deformation and Viscoelastic Behavior of Polymer Gels in Electric Fields, in Neutron Spin Echo Spectroscopy Viscoelasticity Rheology, Springer Berlin Heidelberg, Berlin, Heidelberg, 1997, pp. 131-163.

4 J. P. G. Madden and I. W. Hunter, Conducting polymer actuators as engineering materials, in SPIE's 9th Annual International Symposium on Smart Structures and Materials, SPIE, 2002, p. 15.

5 T. Shoa, J. D. Madden, C. W. E. Fok and T. Mirfakhrai, Rate Limits in Conducting Polymers, Adv. Sci. Technol., 2009, 61, 26-33.

6 R. H. Baughman, Conducting polymer artificial muscles, Synth. Met., 1996, 78(3), 339-353.

7 A. Mazzoldi; A. D. Santa; D. De Rossi, Conducting Polymer Actuators: Properties and Modeling, in Polymer Sensors and Actuators, ed. Osada Y. and De Rossi D. E., Springer Berlin Heidelberg, Berlin, Heidelberg, 2000, pp. 207-244.

8 G. Inzelt, Classification of Electrochemically Active Polymers, in Conducting Polymers: A New Era in Electrochemistry, Springer Berlin Heidelberg, Berlin, Heidelberg, 2008, pp. 7-65.

9 L. Dai, Intelligent Macromolecules for Smart Devices, SpringerVerlag London, 1st edn, 2004.

10 S. K. Goswami, C. J. McAdam, A. M. M. Lee, L. R. Hanton and S. C. Moratti, Linear electrochemical actuators with very large strains using carbon nanotube-redox gel composites, J. Mater. Chem. A, 2013, 1(10), 3415-3420.

11 K. Oyaizu and H. Nishide, Radical Polymers for Organic Electronic Devices: A Radical Departure from Conjugated Polymers?, Adv. Mater., 2009, 21(22), 2339-2344.

12 K. Koshika, N. Sano, K. Oyaizu and H. Nishide, An Aqueous, Electrolyte-Type, Rechargeable Device Utilizing a Hydrophilic Radical Polymer-Cathode, Macromol. Chem. Phys., 2009, 210(22), 1989-1995.

13 T. Suga, K. Yoshimura and H. Nishide, Nitroxide-Substituted Polyether as a New Material for Batteries, Macromol. Symp., 2006, 245-246(1), 416-422.

14 K. Koshika, N. Chikushi, N. Sano, K. Oyaizu and H. Nishide, A TEMPO-substituted polyacrylamide as a new cathode material: an organic rechargeable device composed of polymer electrodes and aqueous electrolyte, Green Chem., 2010, 12(9), 1573-1575.

15 Y. Liang, Z. Tao and J. Chen, Organic Electrode Materials for Rechargeable Lithium Batteries, Adv. Eng. Mater., 2012, 2(7), 742-769.

16 J. R. Fish, S. G. Swarts, M. D. Sevilla and T. Malinski, Electrochemistry and spectroelectrochemistry of nitroxyl free radicals, J. Phys. Chem., 1988, 92(13), 3745-3751.

17 L. Tebben and A. Studer, Nitroxides: Applications in Synthesis and in Polymer Chemistry, Angew. Chem., Int. Ed., 2011, 50(22), 5034-5068.

18 J. M. Bobbitt, Oxoammonium Salts. 6.1 4-Acetylamino2,2,6,6-tetramethylpiperidine-1-oxoammonium Perchlorate: A Stable and Convenient Reagent for the Oxidation of 
Alcohols. Silica Gel Catalysis, J. Org. Chem., 1998, 63(25), 9367-9374.

19 C. M. White, R. Banks, I. Hamerton and J. F. Watts, Characterisation of commercially CVD grown multi-walled carbon nanotubes for paint applications, Prog. Org. Coat., 2016, 90, 44-53.

20 S. R. Malik, F. M. Ruddock, A. H. Dowling, K. Byrne, W. Schmitt, I. Khalakhan, Y. Nemoto, H. Guo, L. K. Shrestha, K. Ariga and J. P. Hill, Graphene composites with dental and biomedical applicability, Beilstein J. Nanotechnol., 2018, 9, 801-808.

21 S. K. Goswami, C. J. McAdam, L. R. Hanton and S. C. Moratti, Hyperelastic Tough Gels through Macrocross-Linking, Macromol. Rapid Commun., 2017, 38(14), 1700103.

22 U. Adash, Synthetic and kinetic investigations into living freen radical polymerisation used in the preparation of polymer therapeutics, $\mathrm{PhD}$ thesis, University of Canterbury, Christchurch, New Zealand, 2005.

23 M. A. Mercadante, C. B. Kelly, J. M. Bobbitt, L. J. Tilley and N. E. Leadbeater, Synthesis of 4-acetamido-2,2,6,6tetramethylpiperidine-1-oxoammonium tetrafluoroborate and 4-acetamido-(2,2,6,6-tetramethyl-piperidin-1-yl)oxyl and their use in oxidative reactions, Nat. Protoc., 2013, 8, 666.
24 A. Ricci, J. Marinello, M. Bortolus, A. Sánchez, A. Grandas, E. Pedroso, Y. Pommier, G. Capranico, A. L. Maniero and G. Zagotto, Electron Paramagnetic Resonance (EPR) Study of Spin-Labeled Camptothecin Derivatives: A Different Look of the Ternary Complex, J. Med. Chem., 2011, 54(4), 1003-1009.

25 T. Suga, H. Ohshiro, S. Sugita, K. Oyaizu and H. Nishide, Emerging N-Type Redox-Active Radical Polymer for a Totally Organic Polymer-Based Rechargeable Battery, Adv. Mater., 2009, 21(16), 1627-1630.

26 L. Rostro, S. H. Wong and B. W. Boudouris, Solid State Electrical Conductivity of Radical Polymers as a Function of Pendant Group Oxidation State, Macromolecules, 2014, 47(11), 3713-3719.

27 L. Dumée, K. Sears, J. Schütz, N. Finn, M. Duke and S. Gray, Influence of the Sonication Temperature on the Debundling Kinetics of Carbon Nanotubes in Propan-2-ol, Nanomaterials, 2013, 3(1), 70-85.

28 H. Abbasi, M. Antunes and J. I. Velasco, Effects of Carbon Nanotubes/Graphene Nanoplatelets Hybrid Systems on the Structure and Properties of Polyetherimide-Based Foams, Polymers, 2018, 10(4), 348. 